\title{
Critical phenomena of thick branes in warped spacetimes
}

\author{
Antonio Campos \\ Institute of Cosmology and Gravitation, University of Portsmouth, Portsmouth PO1 2EG, United Kingdom
}

(Dated: October 27, 2018)

\begin{abstract}
We have investigated the effects of a generic bulk first-order phase transition on thick Minkowski branes in warped geometries. As occurs in Euclidean space, when the system is brought near the phase transition an interface separating two ordered phases splits into two interfaces with a disordered phase in between. A remarkable and distinctive feature is that the critical temperature of the phase transition is lowered due to pure geometrical effects. We have studied a variety of critical exponents and the evolution of the transverse-traceless sector of the metric fluctuations.
\end{abstract}

PACS numbers: $11.10 . \mathrm{Kk}, 04.50 .+\mathrm{h}, 05.70 . \mathrm{Jk}, 11.27 .+\mathrm{d}$

The physics of branes in higher dimensional theories has been the subject of renewed interest in the past years, mostly, because they provide a novel approach for resolving the cosmological constant and the hierarchy problems [1], 2]. An intriguing feature of this type of scenario is the possibility of localizing a massless graviton on the brane, and then reproducing effectively four-dimensional gravity at large distances, due to the warp geometry of the spacetime [3]. Furthermore, these models have also inspired the idea in which our universe is the result of the continuous collisions of branes 四. A fundamental ingredient of this cosmological model is the nucleation and splitting of branes. Then, it is interesting to consider the universal aspects of thick branes [5] splitting in a warped bulk. In this work we present a field theory description for a feasible realization of such a mechanism. Using superpotential techniques [5, 6], some exact solutions of the equations of motion for the coupled scalar field plus gravity system have been studied in [7]. Analytical solutions for giving potentials have been described in [8. The probability of tunneling has been considered in [9] and some geometrical aspects of brane collisions have been pointed out in [10, 11].

In this letter, we provide a qualitative description of the splitting of thick Minkowski branes due to a firstorder phase transition in a warped geometry. For this purpose we have considered a generic potential for a complex scalar field that captures the universal aspects of the phase transition in the bulk. As the system is brought near the critical temperature an interface interpolating two bulk phases breaks into two separated interfaces, and a layer of a new phase appears between them. This effect is known in condensed matter physics as complete wetting [12, 13]. It also appears at the deconfinement phase transition of SU(3) Yang-Mills theory [14, 15] and supersymmetric QCD 16. Our analysis shows the existence of a new effective critical temperature for complete wetting due to the effective negative bulk cosmological constant. This phenomenon reveals a remarkable relationship between geometry and the critical behavior of interfaces. We have been able to characterize the universal aspects of this critical phenomenon computing the critical expo- nents for some relevant quantities. And finally, we have also studied the behavior of the metric fluctuations. In particular, we have found that the zero mode delocalizes, high energy modes are scarcely affected, and for low energy modes the phase changes and the amplitude starts to grow at the moment of the splitting. It is interesting to point out that we are going to use a numerical approach and thereby our solutions do not depend on any specific superpotential.

We start with a metric of the form

$$
d s^{2}=e^{2 A(r)} \eta_{i j} d x^{i} d x^{j}-d r^{2},
$$

where $i=0,1,2,3, \eta_{i j}=(1,-1,-1,-1)$, and the warp factor $A(r)$ depends only on the coordinate of the extra dimension $r$. This choice of metric preserves Poincaré invariance in the four-dimensional submanifolds of constant $r$. To build up the thick brane we consider an auxiliary complex scalar field $\Phi$ coupled to gravity through the five-dimensional action

$S=\int d^{4} x d r \sqrt{|g|}\left(-\frac{1}{4} R+\frac{1}{2} g^{\mu \nu} \partial_{\mu} \Phi^{*} \partial_{\nu} \Phi-V(\Phi)-\Lambda\right)$,

where $R$ is the scalar curvature and $\Lambda$ is a bulk parameter that fixes the minimum of the scalar potential $V(\Phi)$. In flat backgrounds $\Lambda$ is a free parameter that can always be used to set the minimun of the potential to zero. In contrast, this is not always possible if one is concerned to find out soliton solutions of the equations of motion in curved spacetimes. Since we are particularly interested in the universal aspects of first-order phase transitions in the bulk we are going to consider the generic scalar field potential

$$
V(\Phi)=a|\Phi|^{2}-b \phi_{R}\left(\phi_{R}^{2}-3 \phi_{I}^{2}\right)+c|\Phi|^{4},
$$

where $\phi_{R}$ and $\phi_{I}$ are, respectively, the real and the imaginary part of the scalar field $\Phi$. This potential has quartic self-interactions and respects $\mathbb{Z}_{3}$ symmetry. The action for a scalar field with a potential of this type belongs to the same universality class as the effective action for theories describing the $\mathbb{Z}_{N}$ center symmetry of a $S U(N)$ gauge field [15]. So, they can be easily realized if the model supports a bulk field with a gauge 
symmetry. To ensure stability the parameter $c$ must be positive. For $a>0$ the potential has a local minimum at $\Phi^{(1)}=0$ corresponding to a disordered bulk phase. For $b>0$ and $a<9 a_{c} / 8$ there are three additional degenerate global minima corresponding to ordered bulk phases, say $\Phi^{(2)}=\phi_{o}, \Phi^{(3)}=\left(-\frac{1}{2}+i \frac{\sqrt{3}}{2}\right) \phi_{o}$, and $\Phi^{(4)}=\left(-\frac{1}{2}-i \frac{\sqrt{3}}{2}\right) \phi_{o}$, where

$$
\phi_{o}=\frac{3 b}{8 c}\left(1+\sqrt{1-\frac{8}{9} \frac{a}{a_{c}}}\right),
$$

and $a_{c}=b^{2} / 4 c$. These three minima coexist with the disordered phase when $a=a_{c}$. Then fixing $b$ and $c$ lead us to interpret $a$ as a bulk temperature parameter where $a_{c}$ is the critical temperature. The actual variation of $a$ will strongly depend on the particular realization of the effective theory. But, since we are studying the universal aspects of the phase transition, we can treat this parameter as an independent variable. Note that the effective five-dimensional cosmological constant is not $\Lambda$ but $\Lambda_{\text {eff }}=\Lambda+V(a)$, where $V(a)$ indicates the value of the scalar potential at the global minima, i.e. $V\left(\Phi^{(n)}\right) \equiv \phi_{o}^{2}\left(a-b \phi_{o}+c \phi_{o}^{2}\right)$ with $n$ taking values 2,3 or 4.

The classical equations of motion for planar field configurations read as follows [5],

$$
\begin{aligned}
\phi_{R}^{\prime \prime} & =-4 A^{\prime} \phi_{R}^{\prime}+\frac{\partial V}{\partial \phi_{R}}, \\
\phi_{I}^{\prime \prime} & =-4 A^{\prime} \phi_{I}^{\prime}+\frac{\partial V}{\partial \phi_{I}} \\
A^{\prime \prime} & =-\frac{2}{3}\left(\phi_{R}^{\prime 2}+\phi_{I}^{2}\right), \\
3 A^{\prime 2} & =\frac{1}{2}\left(\phi_{R}^{\prime 2}+\phi_{I}^{\prime 2}\right)-V(\Phi)-\Lambda,
\end{aligned}
$$

where the prime denotes differentiation with respect to the coordinate $r$. The soliton equations for the flat case are recovered by taking the warp factor $A(r)$ to be constant 15] (see 16 for a supersymmetric generalization). Note also that Eqs. (6) and (6) imply that $\Lambda<|V(a)|$ and then the effective five-dimensional cosmological constant $\Lambda_{\text {eff }}$ is always negative. We have solved numerically the system of ordinary differential equations (4)(6) for $\phi_{R}(r), \phi_{I}(r)$, and $A(r)$ with boundary conditions $\Phi(-\infty)=\Phi^{(4)}, \Phi(-\infty)=\Phi^{(3)}, A(0)=A^{\prime}(0)=0$ (see Fig. 11). Then, Eq. (7) has been used in order to fix the parameter $\Lambda$ for different values of the parameter $a$ as the interface configuration is brought near the phase transition. Because of the $\mathbb{Z}_{3}$ symmetry there will be equivalent solutions for other choices of ordered phases.

Our results reveals that, equivalently to what happens when the background spacetime is Euclidean, the ordered-ordered domain wall splits into two separated ordered-disordered interfaces, and a layer of disordered phase appears and completely wets the two ordered

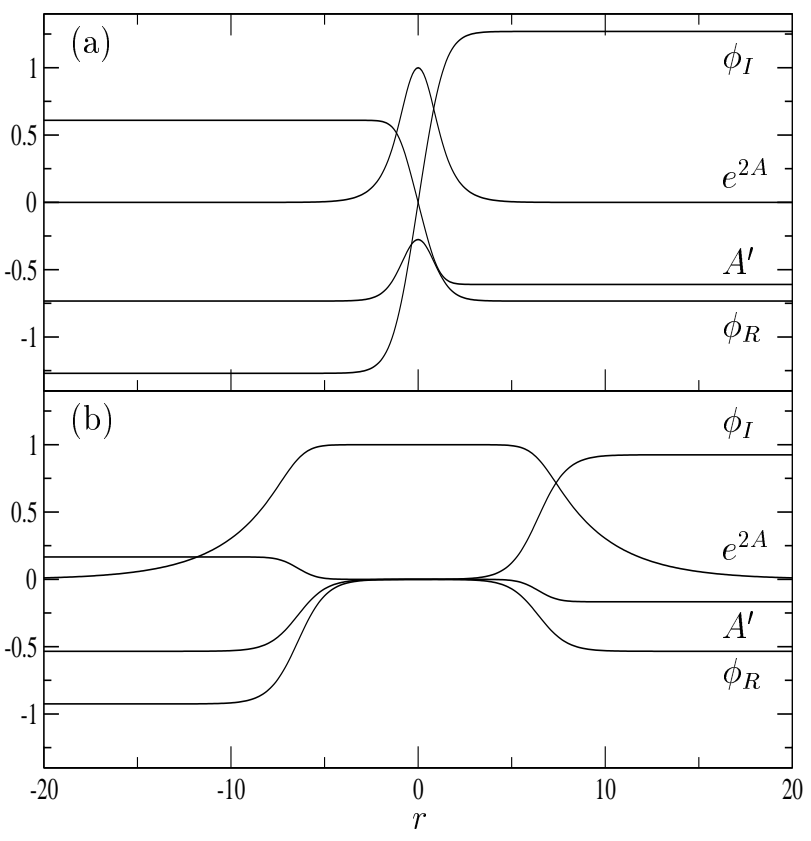

FIG. 1: Interface profiles for thick domain walls in a warped geometry with a $\mathbb{Z}_{3}$-symmetric bulk first-order phase transition for $b=2$ and $c=1$. (a) Away from the phase transition a domain wall interpolates two distinct ordered phases. (b) Close to the critical temperature the wall splits into two interfaces with an intervining film of disordered phase in between.

phases as the the phase transition is approached. Although in both cases this behavior looks quite similar there is an important distinctive feature. For a geometry with a warp factor complete wetting does not occur for the critical temperature $a_{c}$ but for a smaller effective temperature $a_{*}$. As we will see, this effect is closely related to the fact that asymptotically the spacetime is AntideSitter. In order to understand why this effective critical temperature appears it is necessary to know the behavior of the Euclidean energy $E(r) \equiv \frac{1}{2}\left(\phi_{R}^{\prime 2}+\phi_{I}^{\prime 2}\right)-V(\Phi)$ as the temperature parameter $a$ increases. It is very easy to see, using the equations of motion (4) and (5), that the derivative of this energy along the orthogonal direction to the domain wall is $E^{\prime}(r)=-4 A^{\prime}\left(\phi_{R}^{\prime 2}+\phi_{I}^{\prime 2}\right)$. Then, it has a minimun at $r=0, E(0)=\Lambda$, and it reaches its maximun value at infinity $E( \pm \infty)=|V(a)|$. In fact, it has the same shape as $3 A^{\prime 2}$ but is displaced vertically. On the other hand, one also has to take into account that the stability of a configuration with three coexisting phases implies that an interface of two ordered phases can only be completely wet by a disordered phase. This means that in order to have a disordered phase between our initial ordered phases $\Phi(0) \rightarrow \Phi^{(1)}$ and $\Phi^{\prime}(0) \rightarrow 0$ when $a$ increases. Moreover, $E(0)$ should tend to zero as well as $\Lambda$ for complete wetting to occur. If the background is Euclidean, the energy is a positive constant, $E=|V(a)|$, for each value of the temperature parameter $a$. So, since $E$ is zero for $V\left(a=a_{c}\right)=0$, complete wetting happens 
with a critical temperature $a_{c}$. However, in the warped case and because $\Lambda$ is always smaller than $|V(a)|$, the coexistence of the three phases occurs for a critical value $a_{*}<a_{c}$. This inmediately translates into the fact that the effective bulk cosmological constant asymptotically reaches a constant negative value

$$
\Lambda_{e f f}^{*}=\left.V(a)\right|_{a_{*}}<0 .
$$

Then, one could revert the argument and say that the existence of a nonzero negative cosmological constant is responsable for lowering the critical temperature of complete wetting. One would expect intuitively that the value of $a_{*}$ is very close to the critical value $a_{c}$. In this case, it can be easily seen that $\Lambda_{e f f}^{*}, a_{c}$, and $a_{*}$ are related by,

$$
\Lambda_{e f f}^{*} \sim-\frac{a_{c}}{c}\left(a_{c}-a_{*}\right) .
$$

It is worth noticing that the existence of an effective critical temperature prevents the global geometry for becoming flat as the phase transition is reached. If the contrary would have occurred, it would have been a puzzling feature of brane models. In the example plotted in Fig. 1 the critical temperature is $a_{c}=1$. In this case, we have obtained the effective critical temperature $a_{*}=0.9223822740$ for $\Lambda<10^{-7}$ and an effective bulk cosmological constant $\Lambda_{\text {eff }}^{*} \sim-0.08$.

The universal aspects of this critical behavior are characterized by several critical exponents. In Fig. 2 we have plotted the numerical results for the determination of the critical exponents of several parameters of the phase transition. As one would expect from the discussion above, the parameter $\Lambda$ is proportional to the deviation from the effective critical temperature, $\left(a_{*}-a\right) / a_{*} \propto \Lambda$. On the other hand, the width of the disordered phase layer, $r_{o}$, wetting the two ordered phases diverges logarithmically $r_{o} \propto-\log (\Lambda)$. In addition, for the order parameters at the center of the wetting layer we obtain $A^{\prime \prime}(0) \propto \Lambda$, $\phi_{R}(0) \propto \Lambda^{1 / 2}$, and $\phi_{I}^{\prime}(0) \propto \Lambda^{1 / 2}$. In condensed matter physics, this type of critical behaviour corresponds to systems with short-range interactions [13].

Now, let us consider the metric fluctuations with respect to this background solution. In the axial gauge $h_{i 4}=0=h_{44}$ the perturbed metric can be written as

$$
d s^{2}=e^{2 A(r)}\left(\eta_{i j}+h_{i j}\right) d x^{i} d x^{j}-d r^{2} .
$$

The transverse and traceless (TT) sector of the perturbations, $\bar{h}_{i j}$, decouples from the scalar field perturbations and satisfies the equation [5],

$$
\left(\partial_{r}^{2}+4 A^{\prime} \partial_{r}-e^{-2 A} \square\right) \bar{h}_{i j}=0 .
$$

This equation can be transformed into a Schrödinger-like equation by changing to the coordinate $d z=e^{-A} d r$ and introducing the new variable $H_{i j}=e^{-i k x} e^{(3 A / 2)} \bar{h}_{i j}$,

$$
\partial_{z}^{2} H_{i j}=\left(U(z)-k^{2}\right) H_{i j} .
$$

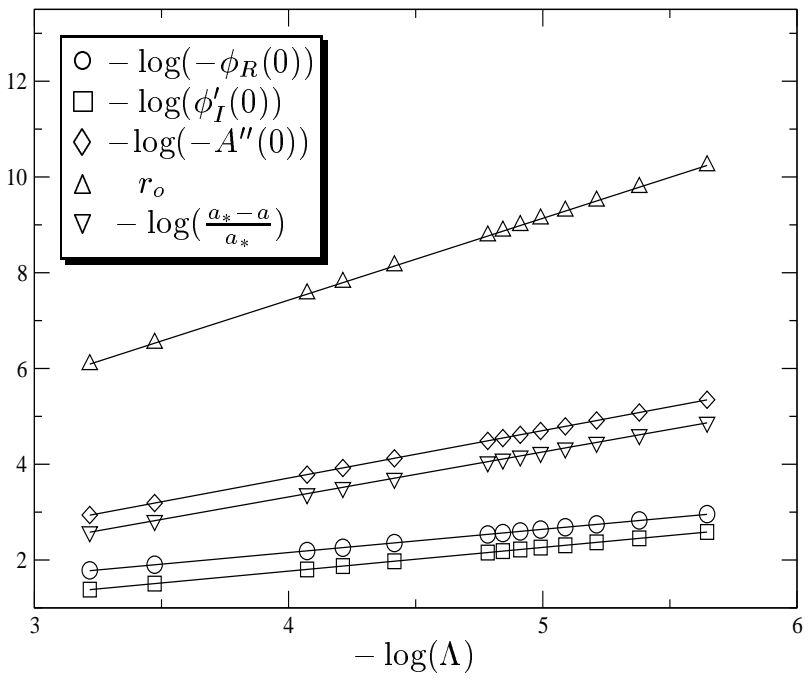

FIG. 2: Determination of the critical exponents for a variety of order parameters of the phase transition.

Here, the potential is

$$
U(z)=\frac{9}{4} \dot{A}^{2}+\frac{3}{2} \ddot{A}=\frac{3}{4} e^{2 A}\left(2 A^{\prime \prime}+5 A^{\prime 2}\right),
$$

where the dot now stands for the derivative with respect to the new coordinate $z$. The zero mode $k=0$ has the analytical solution $H_{i j}=N_{i j} e^{(3 A / 2)}$, with $N_{i j}$ a normalization factor. As shown in Fig. 3 , away from the phase transition the potential in the Schrödinger equation for the TT sector of the metric perturbations has the shape of a volcano potential. However, after the wall splits off, the shape becomes that of a double-well-type potential with a depth decreasing as the two walls separate. One can observe that the critical behavior delocalizes the zero mode, but it does not introduce any gap between the zero mode and the continuum spectrum. Furthermore, the zero mode is the lowest energy eigenstate and then there are no instabilities coming from tachyonic modes.

In Fig. 1, we have also plotted the wave functions of two different types of nonzero modes. In Fig. Q(b), we have considered the case of modes with energy larger than the maximun of the volcano potential, and in Fig. 1(a), those with a smaller energy. The first thing to note is that the wave functions get broader as the mode has lower energy. Modes with larger energy barely get affected by the splitting of the interfaces. On the contrary, the phases of the waves for modes with a small energy change at the moment of the nucleation, increasing their amplitude afterwards.

In conclusion, we have investigated the critical behavior of coexisting interfaces in an asymptotically anti-de Sitter bulk. Because a film of disordered phase is formed between two ordered phases, this mechanism of complete wetting gives a generic field theory description of the splitting of a thick domain wall, which is a fundamental 


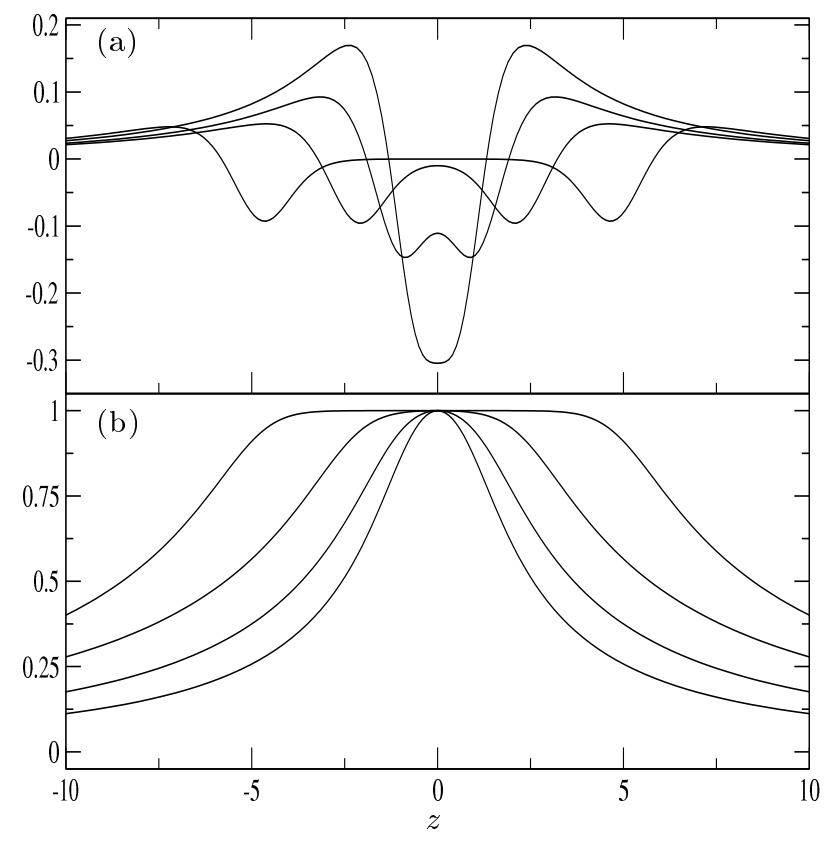

FIG. 3: Shapes for the volcano potential (a) and the wave function of the zeromode (b) for the transverse-traceless part of small gravitational perturbations. The critical temperature is approached from bottom to top in both plots and we have taken an arbitrary normalization for the wave function. Note that, away from the transition, the zeromode is bound to the walls in the fifth extra dimension but, as the walls peel off and pull apart, it is delocalized.

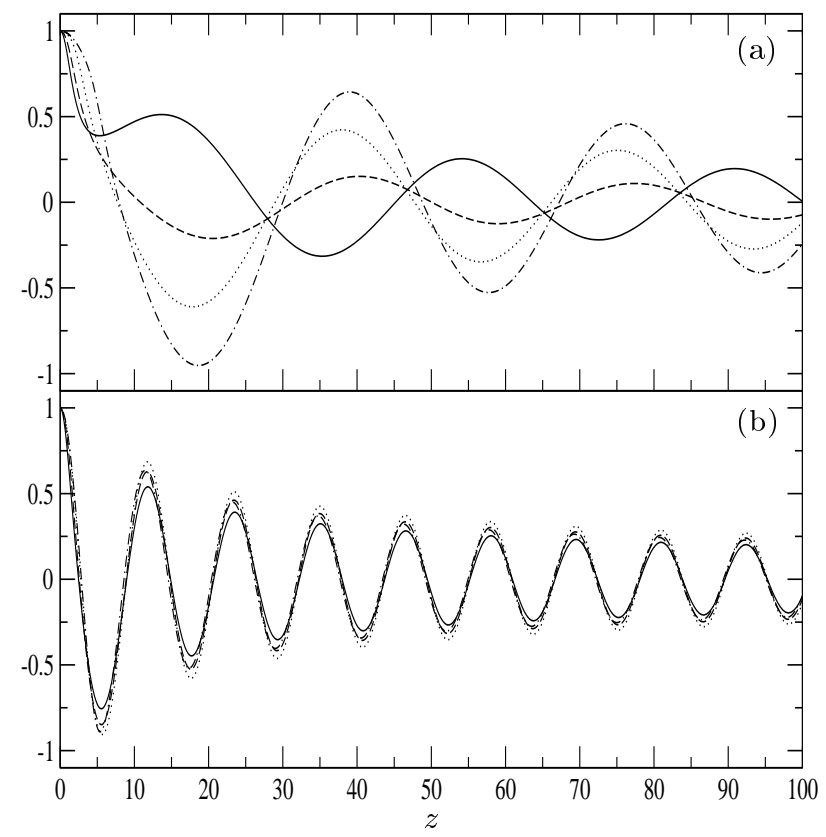

FIG. 4: Wave functions for nonzero modes of metric fluctuations with an arbitrary normalization. (a) Modes with energy smaller than the maximun of the volcano potential, and (b) modes with larger energy. The plots represent different stages of the phase transition in the following order: solid line, dashed line, dot line, and dashed-dot line. Being the latest the closest to the critical temperature. ingredient for the brane cosmological models discussed in [4]. Moreover, we have found that naturally an effective critical temperature shows up due purely to the global geometry. This is a remarkable result because it shows a direct link between geometry and the universal aspects of a critical process. Nevertheless, there still are a few interesting questions that deserve a deeper investigation. For instance, what is the behavior of the non-TT and the scalar field perturbation sectors during the splitting of the thick brane? How does the critical behavior found here change if we consider de Sitter or anti-de Sitter branes instead of Poincaré branes? Is, then, multilocalization of the zero mode possible? Which is the effect of a bulk scalar field?

The author is deeply in debt to C. F. Sopuerta and very thankful to his former collaborators K. Holland and U.-J. Wiese. This work has been supported by the Marie Curie Fellowship HPMF-CT-1999-00158.

[1] N. Arkani-Hamed, S. Dimopoulos, and G. Dvali, Phys. Lett. B 429, 263 (1998).

[2] L. Randall and R. Sundrum, Phys. Rev. Lett. 83, 3370 (1999).

[3] L. Randall and R. Sundrum, Phys. Rev. Lett. 83, 4690 (1999).

[4] J. Khoury, B. A. Ovrut, P. J. Steinhardt, and N. Turok (2001), hep-th/0103239; R. Kallosh, L. Kofman, and A. D. Linde (2001), hep-th/0104073; M. Bucher (2001), hep-th/0107148; U. Gen, A. Ishibashi, and T. Tanaka (2001), hep-th/0110286.

[5] O. DeWolfe, D. Z. Freedman, S. S. Gubser, and A. Karch, Phys. Rev. D62, 046008 (2000).

[6] K. Skenderis and P. K. Townsend, Phys. Lett. B468, 46 (1999); K. Behrndt and M. Cvetič, Phys. Lett. B475, 253 (2000); C. Csáki, J. Erlich, C. Grojean, and T. J. Hollowood, Nucl. Phys. B584, 359 (2000).

[7] M. Gremm, Phys. Lett. B478, 434 (2000); C. Csáki, J. Erlich, T. J. Hollowood, and Y. Shirman, Nucl. Phys. B581, 309 (2000); A. Kehagias and K. Tamvakis, Phys. Lett. B504, 38 (2001); S. Kobayashi, K. Koyama, and J. Soda (2001), hep-th/0107025.

[8] F. Bonjour, C. Charmousis, and R. Gregory, Class. Quant. Grav. 16, 2427 (1999); S. Ichinose, Class. Quant. Grav. 18, 421 (2001); S. Ichinose (2001), hep-th/0107254.

[9] A. Gorsky and K. Selivanov, Phys. Lett. B485, 271 (2000); S. Ng and M. Perry (2001), hep-th/0106008; R. Gregory and A. Padilla (2001), hep-th/0107108.

[10] W. B. Perkins, Phys. Lett. B504, 28 (2001).

[11] A. Neronov (2001), hep-th/0109090; D. Langlois, K. Maeda, and D. Wands (2001), gr-qc/0111013.

[12] R. Lipowsky, Phys. Rev. Lett. 49, 1575 (1982).

[13] R. Lipowsky, Phys. Rev. Lett. 52, 1429 (1984).

14] Z. Frei and A. Patkós, Phys. Lett. B229, 102 (1989).

[15] T. Trappenberg and U.-J. Wiese, Nucl. Phys. B372, 703 (1992).

[16] A. Campos, K. Holland, and U.-J. Wiese, Phys. Rev. Lett. 81, 2420 (1998); ibid., Phys. Lett. B443, 338 (1998); ibid., Nucl. Phys. Proc. Suppl. 73, 438 (1999). 